JAMA. 2009 May 27; 301(20): 2159-2161. doi:10.1001/jama.2009.728.

\title{
Parent-Investigators:
}

\section{A Dilemma}

\author{
David B. Resnik, JD, PhD \\ National Institute of Environmental Health Sciences, National Institutes of Health, Research \\ Triangle Park, North Carolina
}

A recent news story reported that some psychologists and neuroscientists have used their children as study participants. ${ }^{1}$ In one case, a neurology professor had 3 of his children undergo a brain scan with magnetic resonance imaging. In a language development study, a psychologist videotaped $70 \%$ of his son's waking hours for the first 3 years of his life. ${ }^{1}$ Other investigators have also conducted research on their own children, including Edward Jenner, ${ }^{2}$ who tested his smallpox vaccine on his 11 -month-old son; Jonas Salk, who tested his polio vaccine on his own children; and psychologist Jean Piaget, who constructed his theories of child development based on studies of his own children.

Should investigators conduct research on their own children? Federal research regulations ${ }^{3}$ do not address this issue and regulatory agencies, such as the Office of Human Research Protections, ${ }^{4}$ have no policies dealing with it. Other research guidelines, such as the Nuremberg Code $^{5}$ and the Helsinki Declaration, ${ }^{6}$ also do not address the issue of parentinvestigators. When laws and professional codes do not provide guidance concerning human research, investigators and committees that oversee research, such as institutional review boards (IRBs), must rely on their ethical judgment to decide. ${ }^{7}$

\section{Parental Conflict of Interest}

A strong ethical argument can be made that parents should not conduct research on their own children because it creates a conflict of interest that threatens the parent-child relationship and the integrity of science. Parents are responsible for deciding whether their children should participate in research studies and they have moral obligations to support them and protect them from harm. ${ }^{8}$ When parents enroll their own children in a study, their interest in promoting their own research could undermine the obligation to protect their children from harm. For example, parent-investigators might bend inclusion criteria for a study to ensure that their child qualifies, fail to inform the IRB about adverse events or risks, or ignore an older child's objections to participating in a study.

Clinical investigators whose children participate in their research might compromise the integrity of the study to benefit their children. For example, if their children are enrolled in a double-blind randomized clinical trial, parent-investigators might attempt to ascertain whether the children are receiving the experimental treatment, interfere with the randomization, or change the dose of a medication the children are receiving. Clinical investigators who act in this manner might be honoring their obligations as the children's

(C2009 American Medical Association. All rights reserved.

Corresponding Author: David B. Resnick, JD, PhD, NIEHS/NIH, Box 12233 Mail Drop CR 03, Research Triangle Park, NC 27709 (resnikd@niehs.nih.gov).

Financial Disclosure: None reported.

Disclaimer: This Commentary does not represent the views of the NIEHS or NIH. 
physician, but they would be violating legal and ethical rules for the conduct of research. Parent-investigators, in this case, would also succumb to the therapeutic misconception that research studies are specifically designed to benefit research participants. ${ }^{9}$ The temptation to manipulate a research protocol to benefit one's child may also exist in social/behavioral research, but it would occur less frequently than in clinical research because social/ behavioral research usually provides fewer direct benefits for participants. ${ }^{7}$

The conflict of interest that occurs when parents enroll their children in their own research is similar in some ways to the conflict that arises when physicians provide medical treatment for their own children, because in both situations, professional judgment could be affected by personal relationships. ${ }^{10}$ One important difference between physicians who treat their own children and investigators who study their own children is that the goal of medical treatment is to benefit patients, whereas the goal of scientific research is to generate knowledge that can benefit others. ${ }^{11}$ Conducting research on one's own child is therefore potentially more hazardous than treating one's own child because scientific research is not patient centered, and some types of pediatric research impose risks on human participants without the prospect of direct benefit. ${ }^{8}$

\section{Clinical vs Social Behavioral Research}

Some might argue that the ethical argument against participation of children in their parents' research applies only to clinical studies, not to social behavioral research because the risks in social behavioral research are generally much lower in severity than the risks in clinical research. For example, no great harm is likely to result when parents enroll their children in studies in which parent-investigators observe their children's social interactions with other children or ask their children questions about their knowledge of television commercials for a survey. While some clinical studies involve only low-risk procedures or methods, such as collection of biological specimens for biorepositories or physical examinations, many clinical studies involve risky procedures or methods, such as administration of chemotherapy drugs or experimental surgery.

Although the risks of social/behavioral research are generally lower in severity than the risks of clinical research, social/behavioral research is not risk free. Surveys sometimes include questions that are embarrassing or emotionally disturbing. Social/behavioral investigators also deal with sensitive, private information. ${ }^{7}$ Parents may engage in long-term studies of an intrusive nature, such as the project in which the parent videotaped much of his son's first 3 years of life. Moreover, participation of children in their parents' social/behavioral study can strain the parent-child relationship. For example, children may feel some pressure to participate in a study conducted by their mother or father. The children may feel that they cannot refuse to participate or stop participating at any time. Although this pressure also exists when parents who are not the investigator want their child to participate in a study, the pressure can be more intense when parents are the investigators because of the parents' interest in meeting enrollment goals.

Even though the ethical arguments against children participating in their parents' social/ behavioral research are weaker than the arguments against participation in clinical research, there are still some ethical problems with children participating in their parents' social/ behavioral research, and these ethical concerns are sufficient to warrant special attention by oversight committees. Investigators who are planning to enroll their own children in social/ behavioral research should be able to explain to the IRB why their children should be enrolled and how they plan to protect their own children and the integrity of the science.

While there should be a strong presumption against parents conducting clinical research on their own children, there are some exceptions to this rule. First, if parent-investigators are 
not able to determine whether their children are participating in the study, the children's participation would not threaten the parent-child relationship or the integrity of the science. For example, suppose a couple is divorced and the mother is deciding whether her child should participate in a research study that happens to be conducted by the child's father. If the mother does not inform the father about the child's participation, the father's consent is not required, and the father does not interact with research participants or have access to data or samples that personally identify research participants, it could be acceptable for the child to participate.

Second, if a study could provide a child with significant medical benefits, enrolling the child could be justifiable. For example, suppose that a mother, who is a pediatric oncology investigator, has a child who is dying of a rare form of childhood leukemia. Suppose, also, that the mother is conducting the only clinical trial involving a medication with a significant chance of curing the leukemia and that participation in the trial is the best chance of curing the child's cancer or significantly prolonging the child's life. If the child does not participate in the clinical trial, he or she has a high probability of dying within 3 months, but if the child participates in the study, he or she has a chance of living 1 year or more. One could argue that enrollment in this study would be the best option for the child-parental involvement notwithstanding.

Although enrollment of the child in the parent's study might be justified in this case, it is important to be mindful of the potential for therapeutic misconception. The parents should understand that although the study might benefit the child, the main purpose of the study is to develop generalizable knowledge, not to help the child. The parents should understand that the child may not benefit from participation and may experience serious harms. Although one would think that the investigator would not fall prey to the therapeutic misconception, evidence shows that this is often not the case. ${ }^{9}$ Also, additional oversight steps may be needed to protect the integrity of the research and the welfare of the participant. For example, the IRB and data monitoring board should be informed that the researcher is enrolling his or her own child and the noninvestigating parent should also sign the consent, assuming he or she is available.

IRBs should formulate policies addressing the participation of children in their parents' research. The policies should state when investigators may (and may not) enroll their own children in research, when additional monitoring is needed, and address additional protections for children, such as requiring both parents to sign the consent document. IRBs should inform investigators about these policies through seminars, memos, and other educational and training methods.

It may be difficult for IRBs to enforce the policies they develop because IRBs usually do not know the identity of human participants. The documents the IRB reviews, such as protocols, informed consent forms, questionnaires, amendments, adverse event reports, and continuing review forms, do not mention specific research participants. Although the IRB has the authority to examine signed consent forms and other research records, most IRBs do not unless they are auditing a study. ${ }^{7}$ Since IRBs will usually not know whether investigators have their own children participating, IRBs will need to trust investigators to abide by the policies they set. Parents enrolling their own children in their own research must respond appropriately to the dilemma of protecting their children while simultaneously protecting the integrity of the research. It takes special judgment to ensure both are accomplished.

\section{Acknowledgments}

Funding/Support: This research was sponsored by the intramural program of the National Institute of Environmental Health Sciences (NIEHS), National Institutes of Health (NIH). 


\section{References}

1. Belluck, P. New York Times. Jan 19. 2009 Test subjects who call the scientist Mom or Dad; p. A1

2. BBC. [Accessed February 17, 2009] Historic figures: Edward Jenner. 1749-1823. http:// www.bbc.co.uk/history/historic_figures/jenner_edward.shtml

3. US Department of Health and Human Services. [Accessed March 20, 2009] Code of federal regulations: title 45 public welfare; Department of Health and Human Services: part 46 protection of human subjects. http://www.hhs.gov/ohrp/humansubjects/guidance/45cfr46.htm

4. US Department of Health and Human Services. [Accessed March 20, 2009] Office for Human Research Protections: policy guidance (by topics). http://www.hhs.gov/ohrp/policy/index.html

5. National Institutes of Health. Trials of War Criminals Before the Nuremberg Military Tribunals Under Control Council Law No. 10. Vol. 2. Office of Human Subjects Research; Regulations and ethical guidelines, directives for human experimentation: Nuremberg Code; p. 181-182.Web site. http://ohsr.od.nih.gov/guidelines/nuremberg.html [Accessed March 14, 2009]

6. World Medical Association. [Accessed March 14, 2009] Policy: World Medical Association Declaration of Helsinki: ethical principles for medical research involving human subjects. http:// www.wma.net/e/policy/b3.htm

7. Amdur, R.; Bankert, E. Institutional Review Board: Management and Function. 2. Boston, MA: Jones \& Bartlett; 2005.

8. Ross, L. Children in Medical Research: Access Versus Protection. New York, NY: Oxford University Press; 2008.

9. Appelbaum P, Lidz C. Twenty-five years of therapeutic misconception. Hastings Cent Rep. 2008; 38(2):5-6. [PubMed: 18457217]

10. Fromme EK, Farber N, Babbott S, Pickett M, Beasley B. What do you do when your loved one is ill? the line between physician and family member. Ann Intern Med. 2008; 149(11):825-831. [PubMed: 19047031]

11. Levine, RJ. Ethics and Regulation of Clinical Research. 2. New Haven, CT: Yale University Press; 1988. 